\section{A descriptive study of bone density based on angle's malocclusion classification on female patients aged 13-30 years old on panoramic radiograph}

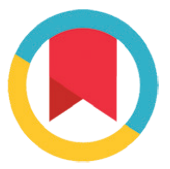

CrossMark

\author{
Pei C. Ling, ${ }^{*}$ Ria N. Firman, Farina Pramanik
}

\title{
Abstract
}

Objective: This study was performed to determine the bone density based on angle's malocclusion classification on female patients aged 13-30 years old using panoramic radiograph.

Material and Methods: Ninety digital panoramic radiographs offemale patients aged 13-30 years old and with 30 radiographs representing each of the angle's malocclusion classes (class I, class II, class III) were used. The bone density was measured by using ImageJ software with 20 x 20 pixels intensity by using a method based on the mental index (MI).
Results: The mean bone density of female patients aged 13-30 years old with angle's malocclusion of class I was $18.726 \%$ of cortical and $81.274 \%$ of marrow, class II was $16.804 \%$ of cortical and $83.196 \%$ of marrow, and class III was $15.911 \%$ of cortical and $84.089 \%$ of marrow. Conclusion: The bone density of female patients aged 13-30 years old with angle's class I malocclusion was higher than class II and class II malocclusion had higher bone density than class III on panoramic radiograph.
Department of Radiology, Faculty of Dentistry, Padjadjaran University, Bandung, Indonesia

*Correspondence to: Pei C. Ling, joyce_ling@hotmail.com

Received: 26 February 2017 Revised: 25 April 2017 Accepted: 21 June 2017 Available online: 01 August 2017

Keywords: Angle's malocclusion classification, Bone density, ImageJ software, Panoramic radiographs

Cite This Article: Ling PC, Firman RN, Pramanik F. 2017. A descriptive study of bone density based on angle's malocclusion classification on female patients aged 13-30 years old on panoramic radiograph. Journal of Dentomaxillofacial Science 2(2): 86-90. D0l:10.15562/bmj.v2i2.523

\section{Introduction}

Malocclusion is one of the most prevailing developmental anomalies of craniofacial structure. According to a national survey done by the Asia Pacific Orthodontic Society (APOS), the malocclusion in Indonesia was above $60 \%$, with class I, then class III and class II. ${ }^{1}$ Although some densitometric studies proposed that the malocclusion had a limited relationship with mandibular bone density, ${ }^{2}$ most of the previous studies proved the other way around. ${ }^{3}$ Individual with dental malocclusion has a more limited masticatory performance due to a few factors including the number and contact areas between maxillary and mandibular teeth, pattern of mastication, and negative effect of occlusal interference. ${ }^{4}$

A dental malocclusion will induce different intensity of mechanical loads on natural teeth or muscles at different regions of the mandible. An increase or decrease of bone density depended on adaptation to these masticatory loads by bone remodeling process. ${ }^{5}$

A close relationship existed between the bone density and the tooth movement. This was because moving the teeth through the bone depended on bone remodeling mechanism and tissue response to orthodontic forces. Therefore, orthodontists needed to take consideration in understanding the bone density in order not to violate the relatively low amount of bony support in the alveolar process. ${ }^{6}$
A digital panoramic radiography was used and image processing program (image) was operated. A previously published data revealed that the suffering due to dental malocclusion was more often in females with $49.0 \%$ than in males with $33.3 \%$. $^{2}$ The current study chose individuals starting from thirteen years old in which angle's malocclusion classification could be used. The age range was chosen only up to thirty-years-old in which most people will reach their peak bone mass.

There were many approaches introduced to study bone density associated with malocclusion over the past few years, ${ }^{2,6}$ but there were no published data about the bone density associated with angle's malocclusion classification. Therefore, the aim of the current study was to evaluate the measurement of bone density of the mandible jaw associated with angle's malocclusion classification on female patients aged 13-30 years old on the panoramic radiograph.

\section{Material and Methods}

The current study was a descriptive study with a cross-sectional method. The sample was 90 digital panoramic radiographs obtained by using stratified random sampling technique from female patients with angle's malocclusion who came to the Dental Hospital Radiology Installation Padjadjaran University. About Ninety samples 
were equally divided among the three classes of angle's malocclusion classification. Each class was then subdivided into three age groups $(1=$ $13-18$ years, $2=19-24$ years and $3=25-30$ years) with 10 patients in each age group.

The secondary data was panoramic radiograph that fulfilled the following criteria: A. Corresponds

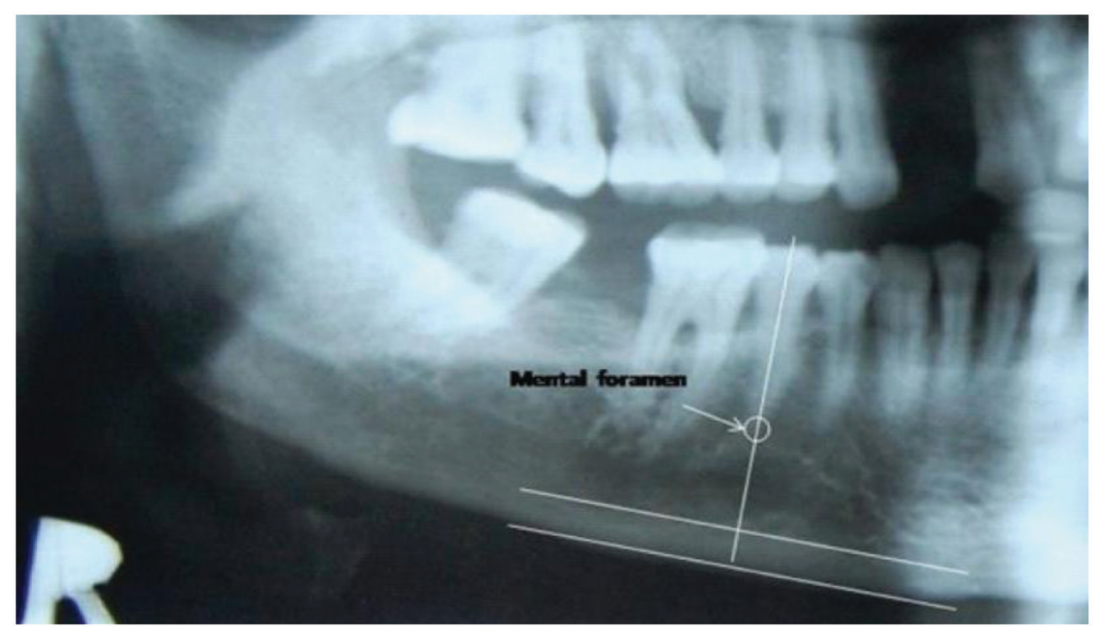

Figure 1 Lines constructed based on MI on panoramic radiograph ${ }^{7}$
A

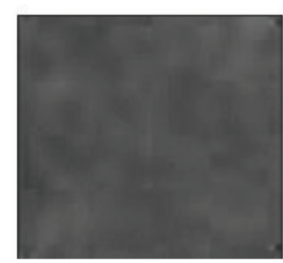

B

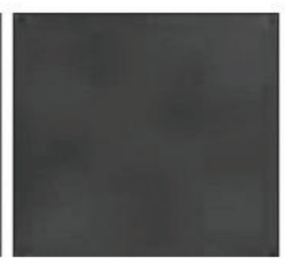

C

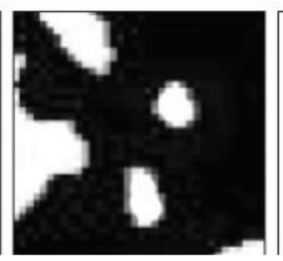

$\mathrm{D}$

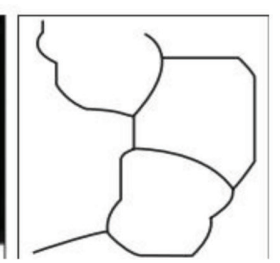

Figure 2 Steps Used in Image Processing. A. Original image, B. Blurred image, C. Binary image, D. Skeletonized image ${ }^{8}$ to the medical record of healthy female patient aged between 13-30 years old with angle's malocclusion classification who had no history of orthodontics treatment and/or orthognathic surgery, B. Illustrates 28 permanent teeth, C. Represents an excellent mandibular bone image, D. Data had not been superimposed in the region of interest and $\mathrm{E}$. Illustrates the absence of any lesions or fractures in the region of interest.

The research protocol was approved by Dental Hospital Faculty of Dentistry Padjadjaran University and Ethics Committee Padjadjaran University. For angle's malocclusion classification, female patients and age range were collected from the medical record in Dental Hospital Padjadjaran University based on the samples' criteria. Then, panoramic radiographs were obtained and screened. The bone density on the panoramic radiograph was the percentage (\%) of cortical and marrow analyzed at the region of interest (ROI) by using ImageJ software application. The ROI was determined based on the MI obtained from panoramic radiograph by drawing a line parallel to the long axis of the mandible and tangential to the inferior border of the mandible, which then intersects with inferior border of the mental foramen and drawing another parallel line at the superior cortical border as shown in figure 1.

The ROI was the area of the distance between the two parallel lines that was the white part of the cortical bone with a square selection tool, it covered 20x20 pixels on both left and right regions of the mandible. The ROI was cropped and duplicated. The duplicated image was blurred with a gaussian filter with sigma 3.00 . Then, the blurred image was subtracted from the original

Table 1 Angle's malocclusion classification of the samples with the corresponding mean values of the percentage of cortical and bone marrow

\begin{tabular}{|c|c|c|c|c|c|c|c|}
\hline \multirow{2}{*}{$\begin{array}{l}\text { Angle's malocclusion } \\
\text { classification }\end{array}$} & \multirow{2}{*}{$\begin{array}{c}\text { Age } \\
\text { (years old) }\end{array}$} & \multicolumn{3}{|c|}{ Percentage of cortical bone (\%) } & \multicolumn{3}{|c|}{ Percentage of bone marrow (\%) } \\
\hline & & Left & Right & Mean & Left & Right & Mean \\
\hline \multirow[t]{4}{*}{ Class I } & $13-18$ & 17.914 & 17.619 & 17.767 & 82.086 & 82.381 & 82.233 \\
\hline & $19-24$ & 17.665 & 18.005 & 17.835 & 82.335 & 81.995 & 82.165 \\
\hline & $25-30$ & 20.000 & 21.156 & 20.578 & 80.000 & 78.844 & 79.422 \\
\hline & Mean & 18.526 & 18.927 & 18.726 & 81.474 & 81.073 & 81.274 \\
\hline \multirow[t]{4}{*}{ Class II } & $13-18$ & 15.692 & 15.813 & 15.752 & 84.308 & 84.187 & 84.248 \\
\hline & $19-24$ & 16.576 & 16.871 & 16.723 & 83.424 & 83.129 & 83.277 \\
\hline & $25-30$ & 17.188 & 18.685 & 17.937 & 82.812 & 81.315 & 82.063 \\
\hline & Mean & 16.485 & 17.123 & 16.804 & 83.515 & 82.877 & 83.196 \\
\hline \multirow[t]{4}{*}{ Class III } & $13-18$ & 15.374 & 16.077 & 15.726 & 84.626 & 83.923 & 84.274 \\
\hline & $19-24$ & 16.191 & 15.669 & 15.930 & 83.809 & 84.331 & 84.070 \\
\hline & $25-30$ & 15.873 & 16.281 & 16.077 & 84.127 & 83.719 & 83.923 \\
\hline & Mean & 15.813 & 16.009 & 15.911 & 84.187 & 83.991 & 84.089 \\
\hline
\end{tabular}




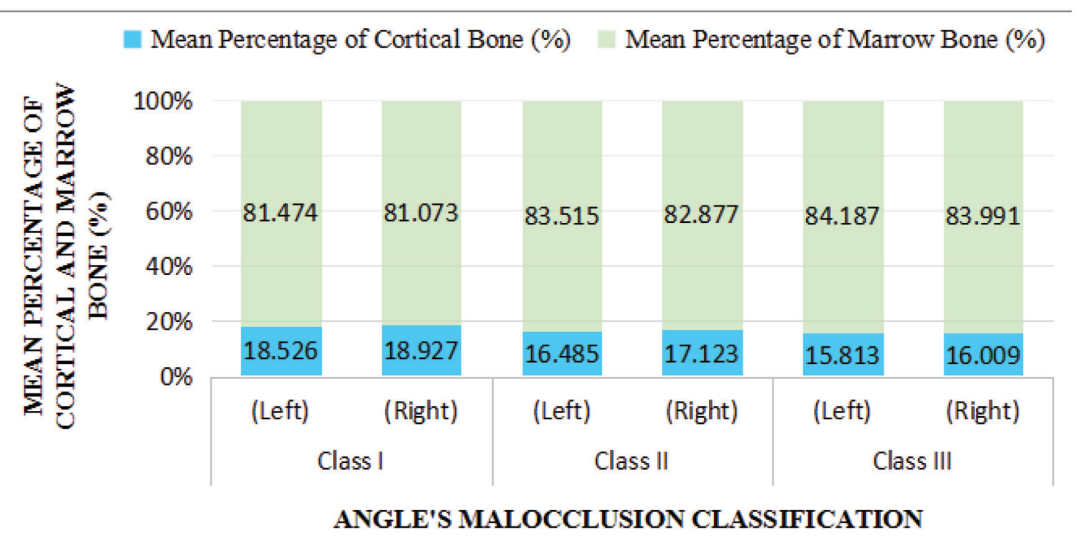

Figure 1 Mean percentages of cortical and bone marrow of the samples with angle's malocclusion classification at the left and right regions

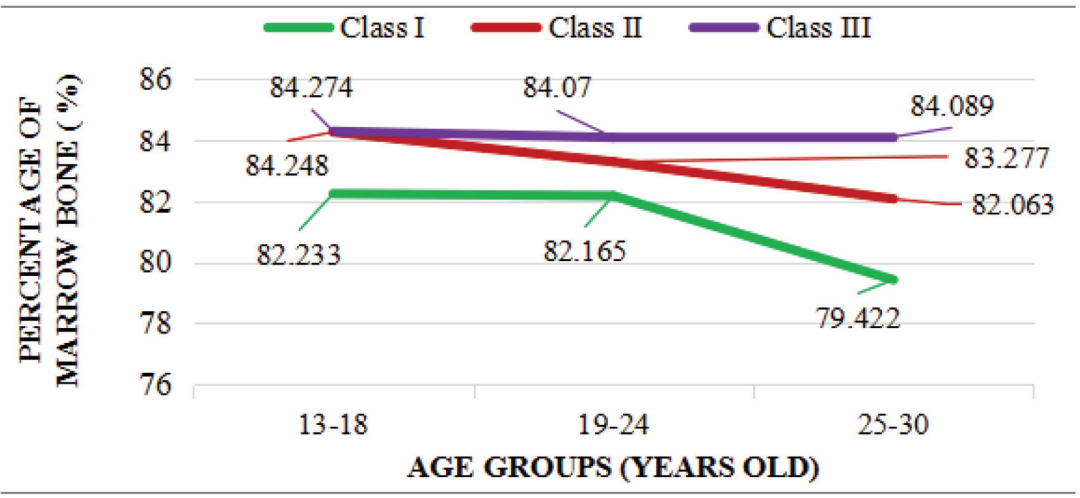

Figure 3 Percentage of bone marrow of the samples against age groups of angle's class I, class II and class III malocclusion

Mean Percentage of Cortical Bone (\%) 1 Mean Percentage of Marrow Bone (\%)

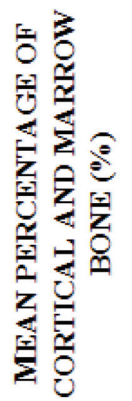

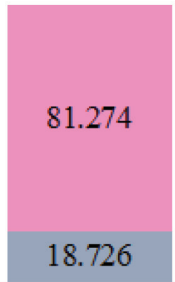

CLASS I

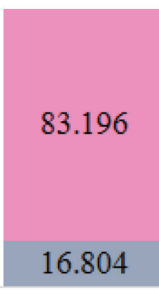

CLASS II

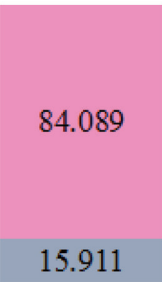

CLASS III

\section{ANGLE'S MALOCCLUSION CLASSIFICATION}

Figure 4 Mean percentages of cortical and bone marrow of the samples against angle's class I, class II and class III malocclusion

image and 128 value was added at each pixel location. The resultant image was converted to binary with a threshold at the grey value of 128 . The binary image was eroded and dilated thrice. Then, the image of the bone was skeletonized. These steps are shown in figure 2. Finally, the ROI was analyzed to determine the percentage of cortical and bone marrow which represented the bone density.

\section{Results}

Each class of angle's malocclusion of the samples was divided into three age groups. Each age group contained 10 samples.

Table 1 showes the mean percentage of cortical bone for angle's class I, II and III malocclusion was $18.726 \%, 16.804 \%$, and $15.911 \%$ respectively. The mean percentage of bone marrow for angle's malocclusion of class I, II and III was $81.274 \%, 83.196 \%$, and $84.089 \%$ respectively.

Based on figure 1, the differences of the percentage of cortical bone at the left region compared to the right region based on angle's malocclusion of class I was $0.401 \%$, class II was $0.638 \%$, and class III was $0.196 \%$. The differences were the same for the percentage of bone marrow as well. Among all the three classes of angle's malocclusion, right regions had higher percentages of cortical bone or lower percentages of bone marrow than the left regions, but the differences of the regions showed nonsignificant values.

Based on figure 2, the percentages of cortical bone for all three classes of angle's malocclusion increased from the lowest age groups to the highest age groups.

Based on all three classes of angle's malocclusion as shown in figure 3 , the percentages of bone marrow decreased from the lowest age groups to the highest age groups.

Figure 4 showed that the angle's class I malocclusion has the highest percentage of cortical bone, followed by class II and lastly was class III. Angle's class III malocclusion has the highest percentage of bone marrow, followed by class II and lastly was class I. This showed about $0.893 \%$ and $2.815 \%$ deficit in the percentage of cortical bone in the mandible for class III malocclusion compared to class II and class I malocclusion respectively. There was a deficit of $1.922 \%$ when the percentage of cortical bone in the mandible for class II malocclusion was compared to class I malocclusion. Similarly, angle's class I malocclusion has a deficit of $1.922 \%$ of bone marrow when compared to class II malocclusion and a deficit of $2.815 \%$ when compared to class III malocclusion. The Angle's class II malocclusion has a deficit of $0.893 \%$ of bone marrow when compared to class III malocclusion.

\section{Discussion}

Female patients aged 13-30 years old with angle's class I malocclusion has the highest bone density, followed by class II and then class III. This was because an individual with angle's class I malocclusion has a higher efficiency of masticatory performance than those with class II malocclusion and the 
poorest masticatory performance was presented in an individual with class III malocclusion. This was in agreement with previous studies. ${ }^{4,9,10}$

A malocclusion could change the physiology of the masticatory system. ${ }^{11}$ Masticatory performance from the stomatognathic system could determine the intensity of mechanical loads through periodontal ligament to the underlying alveolar bone and transferred to the inferior cortical bone of the mandible. ${ }^{2,3,11}$ This physical stimulus can alter the microarchitecture and change the bone density of the mandible. ${ }^{12}$ There were three factors that influenced the masticatory performance. First, the summed number and area of occlusal contacts of antagonistic teeth. Based on angle's classification, ${ }^{10}$ larger particle sizes in patients with malocclusions compared to those without malocclusions was reported. ${ }^{4}$ Compared to normal occlusion, the median particle sizes for class I, class II, and class III malocclusions were roughly $9 \%, 15 \%$ and $34 \%$ larger respectively. ${ }^{10}$

Individuals with angle's class III malocclusions has the smallest contact area with no contact on upper second molars and anterior teeth, indicating the poorest masticatory efficiency, followed by those with class II which might show no occlusal contact on mandibular second molars' regions and anterior teeth as in the class II division 1. The class I malocclusions have better masticatory efficiency although crowding and tooth misalignment can produce a lack of contact with some teeth. ${ }^{10,13,14}$

The second factor was the pattern of mastication. Individual with angle's class I malocclusion has the highest percentage of downward and backward rotation, which indicated a normal masticatory movement. ${ }^{9}$ The percentage of downward and backward rotation was significantly low, in addition, with an erratic rotational direction in an individual with class II malocclusion, which indicated a functionally irregular chewing pattern. An individual with class III malocclusion has an indeterminate rotational direction and there was no or limited excursive movement. ${ }^{13}$ This pattern contributed to a more limited masticatory function than that of class I or class II individuals. ${ }^{14}$

The third factor was the negative effect of occlusal interference, which will induce histological changes in masseter muscles. The occlusal interference caused uncoordinated masticatory muscle activity during mastication in class II and class III individuals. ${ }^{4}$

Individual with class III malocclusion prevented the incisor teeth from performing their functions. The masticatory activity was reduced and this decreased the mechanical loads on the mandibular bone but masticatory difficulty also brought a bigger effort to the masseter and temporalis muscles, creating a higher intensity of stimulus on its bone insertion. Therefore, there was an increase in the volume of the muscle fibers and in consequence, the increase of bone density in regions of insertion of the masseter muscle in the inferior border of mandible., ${ }^{3,11}$ Therefore, samples of female patients with angle's class III malocclusion with the lowest percentage of bone density might be an offset a little by the increase of the volume area of the fiber of the masseter muscles. Therefore, it had only a little bit lower bone density than those with angle's class II malocclusion.

The differences of left and right regions of the percentages of cortical bone or bone marrow of the samples showed insignificant values. These showed a correlation with the results obtained from previous studies. ${ }^{15,16}$

The bone density of all three classes of angle's malocclusion increased from the lowest age groups to the highest age groups. From the age of a newborn, the bone is undergoing growing phase. Although a finding by Snodell et al. ${ }^{17}$ showed that mandibular growth in female continued until the age of eighteen years old, the mandibular bone mass also has a high correlation with the skeletal bone mass. Therefore, the mandibular bone which is closely associated with the growth in stature also increases in bone density until thirty years old which is the peak bone mass. ${ }^{18}$

The current study has several limitations. First, the current study was cross-sectional study. It was unable to fully determine that the samples were not influenced by the environmental factors such as the extent of smoking and alcohol consumption, hormonal levels, nutritional supplements and medication usage that could affect the mandibular bone density. The other limitation was that the race and ethnic origin of the samples were not considered. However, each sample chosen had no obvious low bone density, so it was assumed that the samples were healthy individuals with no influences from the environmental factors and genetic factor.

\section{Conclusion}

Cortical bone has a lower turnover rate than cancellous bone, thus the effect from angle's malocclusion on the cortical bone would be assumed as the sole factor that influenced the bone density. The bone density of female patients aged 13-30 years old with angle's class I malocclusion was higher than class II and class II malocclusion has higher bone density than class III on panoramic radiograph. 


\section{Acknowledgement}

The authors would like to express the deepest appreciation to Prof. Dr. drg. H. Suhardjo, MS., Sp.RKG(K), Department of Radiology Faculty of Dentistry Padjadjaran University for his valuable inputs.

\section{Conflict of Interest}

The authors report no conflict of interest.

\section{References}

1. Tanne Z. Current status of orthodontic professionals in the Asian Pacific region. APOS Trends Orthod 2016;6: 58-77.

2. Konstantynowicz J, Sierpinska T, Kaczmarski M, et al. Dental malocclusion is associated with reduced systemic bone mineral density in adolescents. J Clin Densitom 2007;10: 147-152.

3. Casarin CAS, Bocalini DS, Leite GS, et al. Effect of retrusive displacement of the mandible and increase of the occlusal vertical dimension on mandibular bone density and the masticatory muscles of Wistar rats. Aust J Basic and Appl Sci 2015;9: 711-717.

4. Ngom PI, Diagne F, Aïdara-Tamba AW, et al. Relationship between orthodontic anomalies and masticatory function in adults. Am J Orthod Dentofacial Orthop 2007;131: 216-222.

5. Shokry S, Rahman G, Kandil H, et al. Interdental alveolar bone density in bruxers, mild bruxers and non-bruxers affected by orthodontia and impaction as influencing factors. J Oral Res 2015;4: 378-386.

6. Al-Masri MM, Ajaj MA, Hajeer MY, et al. Evaluation of bone thickness and density in the lower incisors' region in adults with different types of skeletal malocclusion using cone-beam computed tomography. J Contemp Dent Pract 2015; 16: 630-637.

7. Nagi R, Devi Y, Rakesh N, et al. Relationship between femur bone mineral density, body mass index and dental panoramic mandibular cortical width in diagnosis of elderly postmenopausal women with osteoporosis. J Clin Diagn Res 2014;8: 36-41.

8. Kang HJ, Jeong SW, Jo BH, et al. Observation of trabecular changes of the mandible after orthognathic surgery using fractal analysis. J Korean Assoc Oral Maxillofac Surg 2012;38: 96-100.

9. Deguchi T, Garetto L, Sato Y, et al. Statistical analysis of differential lissajous EMG from normal occlusion and class III malocclusion. Angle Orthod 1995;65: 151-160.

10. English JD, Buschang PH, Throckmorton GS. Does malocclusion affect masticatory performance? Angle Orthod 2002;72: 21-27.

11. Casarin CAS, Palma RK, Arsa G, et al. Musculoskeletal analysis of masticatory system arising from malocclusion: literature review. J Morphol Sci 2016;33: 47-50.

12. Koca H, Ergun S, Guneri P, et al. Evaluation of trabecular bone healing by fractal analysis and digital subtraction radiography on digitized panoramic radiographs: a preliminary study. Oral Radiol 2010;26: 1-8.

13. Toro A, Buschang PH, Throckmorton G, et al. Masticatory performance in children and adolescents with class I and II malocclusions. Eur J Orthod 2006;28: 112-119.

14. Vera VR, Ayala AS, Senna PM, et al. Relationship among malocclusion, number of occlusal pairs and mastication. Braz Oral Res 2010;24: 419-424.

15. Schwartz-Dabney CL, Dechow PC. Variations in cortical material properties throughout the human dentate mandible. Am J Phys Anthropol 2003;120: 252-277.

16. Malcic AI, Matijević J, Vodanović $M$, et al. Radiomorphometric indices of mandibular bones in an 18th century population. Arch Oral Biol 2015;60: 730-737.

17. Snodell SF, Nanda RS, Currier GF. A longitudinal cephalometric study of transverse and vertical craniofacial growth. Am J Orthod Dentofacial Orthop 1993;104: 471-483.

18. Rothe LE, Bollen AM, Little RM, et al. Trabecular and cortical bone as risk factors for orthodontic relapse. Am J Orthod Dentofacial Orthop 2016;130: 476-484.

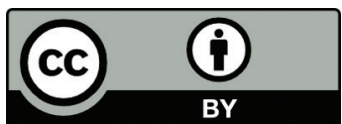

This work is licensed under a Creative Commons Attribution 